\section{Schmerztherapie nach Knieersatz: Lokalanästhesie so gut wie Femoralisblock}

\section{Lokale Infiltrationsanalgesie lindert die Schmerzen nach Implantation einer Totalendoprothese (TEP) am Knie ebenso gut wie eine Femoralisblockade. Dies haben Anästhesisten und Orthopäden der schwedischen Karolinska Universitätsklinik in einer Studie mit 40 Patienten gezeigt.}

$\mathrm{D}$ er Operation zum Knieersatz, die jeweils in Spinalanästhesie erfolgte, mussten sich die Patienten wegen Arthrose oder rheumatoider Arthritis unterziehen. Vor dem Eingriff wurden sie einer von zwei Gruppen zugelost. Die 20 Probanden der Gruppe 1 erhielten direkt nach der Spinalanästhesie eine Femoralisblockade, wobei über einen Katheter 24 Stunden lang vierstündlich Ropivacain in die Umgebung de Nervus femoralis gespritzt wurde. Zusätzlich bekamen sie achtstündliche Ketorolac-Injektionen i.v.

Bei den 20 Patienten der Gruppe 2 injizierten die Chirurgen lokal eine Lösung aus Ropivacain, Ketorolac und Epinephrin, und zwar zu Beginn der OP intrakutan sowie während des Eingriffs in die hintere Gelenkkapsel, in die Nähe der Inzision, in die Musculi vastus intermedius et lateralis und in die Seitenbänsteuern konnten. der. Eine dritte Dosis wurde den Patienten am Ende des Eingriffs über einen intraartikulären Katheter verabreicht. Postoperativ waren alle Patienten mit einer Morphinpumpe versorgt,über die sie ihre Analgesie bedarfsgerecht selbst

Die durchschnittliche Schmerzintensität, angegeben auf einer numerischen Bewertungsskala von 0 (keine Schmerzen) bis 10 (schlimmste vorstellbare Schmerzen), war in Gruppe 2 mit 1,6 etwas geringer als in Gruppe 1, wo der Durchschnittswert bei 2,2 lag. Auch der Morphinverbrauch unterschied sich nicht signifikant. Eine Nebenanalyse ergab, dass in der Gruppe mit Lokalanästhesie ein Patient über eine Schmerzstärke $>7$ bei Bewegung berichtete, in der Femoralisblock-Gruppe waren es sieben. Dieser Unterschied war zwar signifikant $(\mathrm{p}=0,04)$, müsste als Ergebnis einer $\mathrm{Zu}$ -

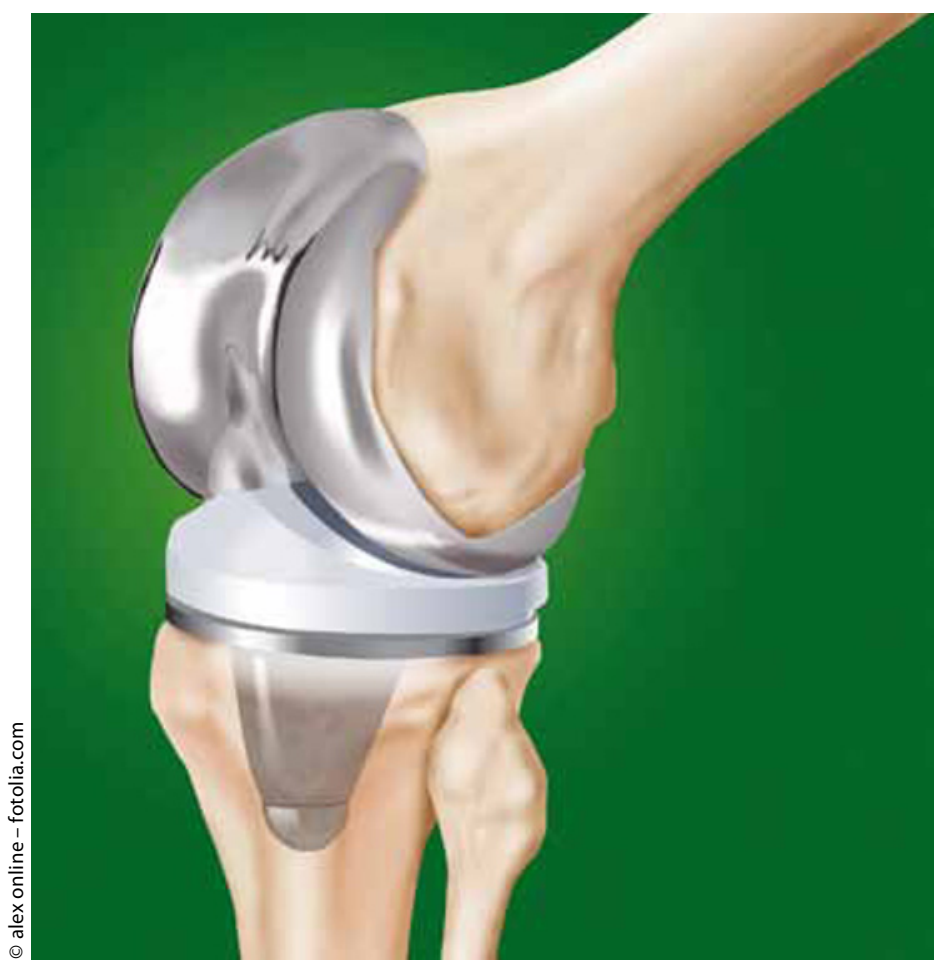

Nach Knie-TEP treten üblicherweise starke Schmerzen auf. Zur Analgesie wird traditionell die Femoralisblockade eingesetzt - und die könnte nun lokale Konkurrenz bekommen. satzanalyse aber in einer eigenen Studie bestätigt werden.

Fazit: In der vorliegenden Studie erzielte nach Knie-TEP die Analgesie mithilfe lokaler Infiltrationsanästhesie eine ebenso gute - tendenziell sogar bessere - Schmerzkontrolle wie eine Femoralisblockade. Möglicherweise erreicht die Infiltrationsanalgesie auch Gewebestrukturen, die nicht vom Femoralis-, sondern vom Ischiasnerv innerviert werden. Die lokale Analgesie ist zudem billiger und technisch einfacher $\mathrm{zu}$ handhaben als der Femoralisblock.

Dr. Robert Bublak

Affas F et al. Pain control after total knee arthroplasty: a randomized trial comparing local infiltration anesthesia and continuous femoral block. Acta Orthopaedica 2011:82:441-7

Kommentar: Die Ergebnisse der Studie von Affas et al. besitzen für jeden Endoprothetiker höchste Relevanz. Die Möglichkeit einer schmerzfreien, unkomplizierten und kostengünstigen Frühmobilisation sind sowohl bezüglich der Patientenzufriedenheit als auch unter ökonomischen Gesichtspunkten höchst interessant. Die Autoren zeigen, dass die intra- und periartikuläre Injektion eines Cocktails bestehend aus Lokalanästhetikum, NSAR und Adrenalin während der Operation der Femoralisblockade per Schmerzkatheter mindestens ebenbürtig ist, um postoperative Schmerzen zu minimieren. Die potenziellen Vorteile dieser Methode beinhalten unter anderem eine deutlich einfachere Handhabung und Nachsorge sowie ein geringeres Risiko unerwünschter Nebenwirkungen. 